\title{
Mechanisms behind the superior effects of interval vs continuous training on glycaemic control in individuals with type 2 diabetes: a randomised controlled trial
}

\author{
Kristian Karstoft • Kamilla Winding • Sine H. Knudsen • \\ Noemi G. James • Maria M. Scheel • Jesper Olesen • \\ Jens J. Holst • Bente K. Pedersen • Thomas P. J. Solomon
}

Received: 17 May 2014 / Accepted: 30 June 2014 / Published online: 7 August 2014

(C) Springer-Verlag Berlin Heidelberg 2014

\begin{abstract}
Aims/hypothesis By use of a parallel and partly crossover randomised, controlled trial design we sought to elucidate the underlying mechanisms behind the advantageous effects of interval walking training (IWT) compared with continuous walking training (CWT) on glycaemic control in individuals with type 2 diabetes. We hypothesised that IWT, more than CWT, would improve insulin sensitivity including skeletal muscle insulin signalling, insulin secretion and disposition index (DI).
\end{abstract}

Electronic supplementary material The online version of this article (doi:10.1007/s00125-014-3334-5) contains peer-reviewed but unedited supplementary material, which is available to authorised users.

K. Karstoft $\cdot$ K. Winding $\cdot$ S. H. Knudsen $\cdot$ N. G. James

M. M. Scheel • J. Olesen • B. K. Pedersen • T. P. J. Solomon

The Centre of Inflammation and Metabolism, Department of Infectious Diseases and CMRC, Rigshospitalet, Faculty of Health

Sciences, University of Copenhagen, Copenhagen, Denmark

K. Karstoft $\cdot$ K. Winding $\cdot$ S. H. Knudsen $\cdot$ N. G. James $•$

M. M. Scheel · J. Olesen • B. K. Pedersen • T. P. J. Solomon

The Centre for Physical Activity Research, Department of Infectious

Diseases and CMRC, Rigshospitalet, Faculty of Health Sciences,

University of Copenhagen, Copenhagen, Denmark

J. Olesen

Department of Biology, University of Copenhagen,

Copenhagen, Denmark

J. J. Holst • T. P. J. Solomon ( $\square)$

Department of Biomedical Sciences, University of Copenhagen,

Blegdamsvej 3B, Panum Institute 4.5.13,

DK-2200 Copenhagen, Denmark

e-mail: thomas.solomon@sund.ku.dk

J. J. Holst

Novo Nordisk Foundation Centre for Basic Metabolic Research, Panum Institute, University of Copenhagen, Copenhagen, Denmark
Methods By simple randomisation (sequentially numbered, opaque sealed envelopes), eligible individuals (diagnosed with type 2 diabetes, no exogenous insulin treatment) were allocated to three groups: a control group (CON, $n=8)$, an IWT group $(n=12)$ and an energy expenditure-matched CWT group $(n=12)$. Training groups were prescribed free-living training, five sessions per week (60 min/session). A threestage hyperglycaemic clamp, including glucose isotope tracers and skeletal muscle biopsies, was performed before and after a 4 month intervention in a hospitalised setting. No blinding was performed.

Results The improved glycaemic control, which was only seen in the IWT group, was consistent with IWT-induced increases in insulin sensitivity index $(49.8 \pm 14.6 \%$; $p<0.001)$, peripheral glucose disposal $(14.5 \pm 4.9 \%$; $p<0.05)$ and DI $(66.2 \pm 21.8 \% ; p<0.001)$, with no changes in the CWT or CON group. Moreover, only IWT improved insulin signalling in skeletal muscle via increased insulin-stimulated phosphorylation of AS160 $(29.0 \pm 10.8 \% ; p<0.05)$. No changes were seen in insulin secretion during hyperglycaemia alone, hyperglycaemia + glucagon-like peptide 1 infusion or arginine injection.

Conclusions/interpretation IWT maintains insulin secretion and improves insulin sensitivity and DI, in contrast to energy expenditure-matched CWT. These results suggest that training with alternating intensity, and not just training volume and mean intensity, is a key determinant of changes in whole body glucose disposal in individuals with type 2 diabetes. Trial registration: ClinicalTrials (NCT01234155).

Keywords Beta cell function · Endurance training - Exercise · Glycaemic control · Hyperglycaemic clamp · Insulin secretion · Insulin sensitivity · Insulin signalling · Physical activity · Type 2 diabetes 


$\begin{array}{ll}\text { Abbreviations } \\ \text { CON } & \text { Control } \\ \text { CS } & \text { Citrate synthase } \\ \text { CWT } & \text { Continuous walking training } \\ \text { DI } & \text { Disposition index } \\ \text { GIR } & \text { Glucose infusion rate } \\ \text { GLP-1 } & \text { Glucagon-like peptide 1 } \\ \text { GSK } & \text { Glycogen synthase kinase } \\ \text { ISR } & \text { Insulin secretion rates } \\ \text { IWT } & \text { Interval walking training } \\ \text { PGC-1 } \alpha & \text { Peroxisome proliferator-activated receptor } \\ & \text { gamma coactivator 1 } \alpha \\ R_{\mathrm{a}} & \text { Endogenous rate of appearance } \\ R_{\mathrm{d}} & \text { Rate of disappearance } \\ \mathrm{S}_{\mathrm{I}} & \text { Insulin sensitivity index } \\ \dot{V} \mathrm{O}_{2 \text { max }} & \text { Maximal energy expenditure rate for walking } \\ \dot{V} \mathrm{O}_{2 \text { peak }} & \text { Peak energy expenditure rate for walking } \\ \mathrm{wW} & \text { Wet weight }\end{array}$

\section{Introduction}

The effects of exercise on glycaemic control in individuals with type 2 diabetes are well documented, but the optimal exercise intensity and type remain to be defined. Traditionally, high-intensity exercise has not been recommended for individuals with type 2 diabetes due to a fear of inducing injuries and lowering adherence [1]. Nevertheless, high-intensity exercise improves glycaemic control more than low-intensity exercise [2]. During interval training (training with alternating intensities), peak exercise intensity is increased, which theoretically should be advantageous for improving glycaemic control [3], and recent studies of interval training programmes have shown remarkable results on glycaemic control $[4,5]$. In this context, we have shown that interval walking training (IWT) more favourably improves glycaemic control in type 2 diabetic individuals when compared with energy expenditurematched continuous walking training (CWT) [6].

Glycaemic control is primarily determined by insulin sensitivity and pancreatic endocrine function, both of which are impaired in individuals with type 2 diabetes. It is well accepted that physical activity improves insulin sensitivity $[7,8]$, but the underlying mechanisms are more controversial [9]. The effect of exercise intensity on insulin sensitivity is debated [10-13], and whether it is differentially affected by interval vs well-matched continuous exercise in individuals with type 2 diabetes is unknown. Rodent studies indicate that both lowand high-intensity exercise result in improvements in insulin sensitivity, but potentially via different mechanisms $[14,15]$. If these data are translatable to humans, interval training may be the optimal approach for type 2 diabetic individuals.
Insulin secretion has been shown to increase [16, 17], decrease [18] or remain unchanged [19] following training interventions in individuals with type 2 diabetes, and the impact of interval training is unknown. Since insulin secretion is a better predictor of training-induced changes in glycaemic control compared with insulin sensitivity [20], it is of great relevance. Moreover, the disposition index (DI), a marker of pancreatic beta cell function adjusted for insulin sensitivity, is tightly correlated to glycaemic control [21]. While the Studies of a Targeted Risk Reduction Intervention through Defined Exercise (STRRIDE) trial demonstrated that exercise intensity is important for training-induced changes in DI in healthy individuals [22], it is unknown whether this is also true in individuals with type 2 diabetes.

The aim of the present study was to elucidate the underlying mechanisms behind the advantageous effects of interval training compared with continuous training on glycaemic control, in individuals with type 2 diabetes. An in-depth characterisation of these mechanisms, comparing well-matched training interventions, has never been performed. We hypothesised that interval training, compared with continuous training, would better improve insulin sensitivity, insulin secretion and DI.

\section{Methods}

Individuals with type 2 diabetes [23] underwent a medical screening test from August 2010. Exclusion criteria were: use of exogenous insulin; weight instability ( $>2 \mathrm{~kg} / 6$ months); high levels of physical activity ( $>150 \mathrm{~min} /$ week); evidence of liver, renal or cardiopulmonary disease or of diseases contraindicating physical activity [24]. Individuals included in the study were randomised by the investigators to three groups (by picking an envelope with predefined group numbers): a control group (CON), a CWT group and an IWT group. Since five individuals initially randomised to the CON group were re-randomised to either IWT or CWT after the CON period, the final study population consisted of $n=27$ individuals with $n=32$ pre-/post-intervention trial participants included for statistical analysis (CON, $n=8$; CWT, $n=12$; IWT, $n=12$ ). The randomisation procedure and the body composition, physical fitness and glycaemic control data have been reported in full elsewhere [6]. Written informed consent was obtained from all individuals. The study was approved by the ethics committee of the Capital Region of Denmark and registered at www.ClinicalTrials.gov (NCT01234155).

Interventions

All individuals received a training device (JD Mate; Kissei Comtec, Matsumoto, Japan) [25] which was used to monitor daily physical activity (steps) throughout the study. 
Additionally, individuals randomised to a training group used the JD Mate's training function which, based on tri-axial accelerometry, estimates training-derived energy expenditure [26]. Furthermore, all training sessions were performed with a heart rate monitor (Polar RS400; Polar, Kempele, Finland). The training protocol has been described in detail elsewhere [6]. Briefly, CWT consisted of continuous walking at individualised moderate intensity ( $\geq 55 \%$ of the individual's peak energy expenditure rate for walking $\left[\dot{V} \mathrm{O}_{2 \text { peak }}\right]$ ), whereas IWT consisted of alternating 3 min intervals of fast $(\geq 70 \%$ of $\left.\dot{V} \mathrm{O}_{2 \text { peak }}\right)$ and slow ( $\sim 40 \%$ of $\left.\dot{V} \mathrm{O}_{2 \text { peak }}\right)$ walking. IWT individuals were instructed in these cycles by the JD Mate via audible signals. The aim was to match CWT and IWT by overall energy expenditure and mean training intensity. All training individuals were prescribed five free-living training sessions/ week, $60 \mathrm{~min} / \mathrm{session}$ for 4 months, and training effort was monitored by data upload every second week. Individuals in the CON group were instructed to continue their habitual lifestyle for 4 months and had their physical activity data uploaded monthly.

\section{Investigations}

Prior to the pre-intervention tests, individuals abstained from their glucose-lowering, antihypertensive and lipid-lowering drugs for 1 week. On day 1, following an overnight fast ( $\geq 8 \mathrm{~h}$ ), an antecubital venous catheter was placed and a $3 \mathrm{~h}$ $75 \mathrm{~g}$ OGTT was performed as previously described [6]. On day 2 , following an overnight fast ( $\geq 8 \mathrm{~h}$ ), a three-stage hyperglycaemic clamp was performed. An antecubital venous catheter was placed for infusions and a retrograde venous catheter was placed in the contralateral hand for blood sampling. The latter was kept warm using a heating blanket $\left(\sim 60^{\circ} \mathrm{C}\right)$. After a baseline blood sample, a primed $(20 \mu \mathrm{mol} / \mathrm{kg}$ multiplied by fasting glucose divided by $5 \mathrm{mmol} / \mathrm{l})$, continuous $\left(0.2 \mu \mathrm{mol} \mathrm{kg}{ }^{-1} \mathrm{~min}^{-1}\right)\left[6,6-{ }^{2} \mathrm{H}_{2}\right]$ glucose infusion began $(t=0)$. At $t=120 \mathrm{~min}$, the $\left[6,6-{ }^{2} \mathrm{H}_{2}\right]$ glucose infusion was tripled

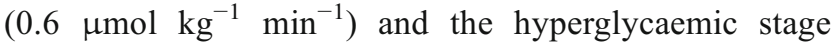
commenced: a square-wave $20 \%$ (wt/vol.) glucose infusion began, aiming to increase baseline glucose concentrations by $5.4 \mathrm{mmol} / 1$ [27]. Blood glucose was clamped by adjusting the glucose infusion rate (GIR), based on glucose measurements obtained every 5 min (ABL 7 series; Radiometer, Herlev, Denmark). GIR was changed using a computerised algorithm based on the original description by DeFronzo et al [28]. At $t=240 \mathrm{~min}$, the hyperglycaemic + glucagon-like peptide 1 (GLP-1) stage commenced: a primed $(0.2 \mathrm{pmol} / \mathrm{kg})$, continuous $\left(0.5 \mathrm{pmol} \mathrm{kg}^{-1} \mathrm{~min}^{-1}\right)$ infusion of GLP-1 mimicking postprandial concentrations in healthy individuals [29] began. At $t=300 \mathrm{~min}$, the arginine stage commenced: an injection of arginine hydrochloride ( 5 g given over $30 \mathrm{~s}$ ) was administered to assess maximal insulin secretory capacity. Glucose and
GLP-1 infusion was continued until $t=330 \mathrm{~min}$, when the clamp was terminated. Blood samples for insulin and C-peptide measurements (serum tubes) were obtained at 1 , 2, 4 and $6 \mathrm{~min}$ into each stage (acute-phase insulin secretion) and every $10 \mathrm{~min}$ during the last $30 \mathrm{~min}$ of the basal, hyperglycaemic and hyperglycaemic + GLP-1 stages (late-phase insulin secretion). Blood samples for glucose tracer enrichment analyses ( $\mathrm{NaF}$ tubes) were also obtained during these late phases. At the end of each stage, blood samples were obtained for measurement of glucagon (EDTA tubes) and GLP-1 (EDTA tubes + aprotinin [500 kU/ml Trasylol; Bayer HealthCare, Berlin, Germany] and dipeptidyl peptidase-4 inhibitor [0.01 mmol/1 diprotin A; Sigma, Copenhagen, Denmark]). Plasma samples were placed on ice and centrifuged $\left(2,000 \mathrm{~g}, 15 \mathrm{~min}, 4^{\circ} \mathrm{C}\right)$. Serum samples were left at room temperature for $30 \mathrm{~min}$ before centrifugation. Muscle biopsies were obtained at baseline and at the end of the hyperglycaemic stage from vastus lateralis in the quadriceps femoris muscle [30]. Muscle tissue was immediately frozen in liquid nitrogen. Samples were stored at $-80^{\circ} \mathrm{C}$ until analysis.

A repeated measure of the pre-intervention tests was performed after the intervention period. To avoid the effects of acute exercise, and since other variables were also assessed [6], the OGTT was performed 5-6 days after the last exercise bout, whereas the hyperglycaemic clamp was performed the day after the OGTT. Each individual was clamped at the same glucose levels pre- and post-intervention.

\section{Blood chemistry analyses}

Glucose tracer analyses were performed by a liquidchromatography-mass-spectrometry hexabenzoyl derivative method [31]. Endogenous rate of appearance $\left(R_{\mathrm{a}}\right)$ and rate of disappearance $\left(R_{\mathrm{d}}\right)$ were derived using non-steady-state assumptions [32]. Insulin and C-peptide were measured by an electrochemiluminescence immunoassay (E-modular; Roche, Basel, Switzerland). Glucagon was measured by RIA (EMD Millipore, Merck KGaA, Darmstadt, Germany) according to the manufacturer's protocols. Total GLP-1 was measured by RIA (antisera 89390) specific for the $\mathrm{COOH}$ terminus of the GLP-1 molecule and thus detecting intact GLP-1 and the primary (NH2 terminally truncated) metabolite [33].

Skeletal muscle analyses

Glycogen content Tissue ( 5-10 mg wet weight [ww]) was boiled for $2 \mathrm{~h}$ in $500 \mu \mathrm{l} 2 \mathrm{~mol} / \mathrm{l} \mathrm{HCl}$. Following neutralisation (2 mol/1 NaOH), free glucose was quantified (Amplex Red Glucose Assay; Thermo Fisher Scientific, Waltham, MA, USA).

PCR analyses Tissue ( $15 \mathrm{mg}$ ww) was homogenised (Tissuelyser; Qiagen, Hilden, Germany) in TRIzol (Thermo 
Fisher Scientific). RNA was isolated and quantified (Nanodrop ND 1000; Thermo Fisher Scientific). RNA $(0.5 \mu \mathrm{g})$ was reverse transcribed (cDNA high-capacity kit; Applied Biosystems, Foster City, CA, USA). cDNA samples were diluted in nuclease-free water (total volume $200 \mu \mathrm{l}$ ). Real-time quantitative PCR was performed (ViiA 7; Applied Biosystems), with samples determined in triplicate. Primer and TaqMan probe sequences are provided in Table 1 of the electronic supplementary material (ESM). A pre-optimised assay (Applied Biosystems) for 18S rRNA was used for normalisation.

Western blotting Tissue ( $30 \mathrm{mg}$ ww) was homogenised (Tissuelyser; Qiagen) in $50 \mathrm{mmol} / \mathrm{l}$ Tris- $\mathrm{HCl}, \mathrm{pH} \mathrm{7.4,}$ $150 \mathrm{mmol} / 1 \mathrm{NaCl}, 1 \mathrm{mmol} / 1 \mathrm{EGTA}, 1 \mathrm{mmol} / 1$ EDTA, $50 \mathrm{mmol} / \mathrm{l} \mathrm{NaF}$ and $50 \mathrm{mmol} / \mathrm{l} \mathrm{NaP}$. Phosphatase inhibitor cocktail 2+3 (Sigma-Aldrich, St. Louis, MO, USA), protease inhibitor Complete Mini (Roche), NaV 20 mmol/l, dithiothreitol (DTT) $10 \mathrm{mmol} / 1$ and $0.2 \%$ IGEPAL-CA-630 was added. Protein concentration was measured (colorimetric assay; BioRad, Hercules, CA, USA). Loading buffer (Thermo Fisher Scientific) and $100 \mathrm{mmol} / \mathrm{l}$ DTT was added and samples boiled for $5 \mathrm{~min}$ (samples for GLUT4 measurements were not boiled). Lysate $20 \mu \mathrm{g}$ was loaded (Criterion TGX 4-15\% gel; Bio-Rad). All samples from one individual were loaded on the same gel. Gels were run at $180 \mathrm{~V}$ for $50 \mathrm{~min}$. Protein was transferred onto a polyvinylidene fluoride membrane using the Trans-Blot Turbo System (Bio-Rad) at $25 \mathrm{~V}$ for $10 \mathrm{~min}$. Membranes were blocked $(1 \mathrm{~h})$ and incubated with primary antibody overnight $\left(4^{\circ} \mathrm{C}\right)$. Primary antibodies and dilutions are shown in ESM Table 2. Membranes were washed and incubated with secondary horseradish peroxidaseconjugated IgG antibody (Dako, Glostrup, Denmark). Signal was detected (Supersignal West Femto Luminal/Enhancer Solution; Thermo Fisher Scientific) and exposed (chargecoupled camera; Bio-Rad). For normalisation, membranes were incubated in $0.5 \%$ Reactive Brown (Sigma) for $15 \mathrm{~min}$. Blots were analysed and quantified using ImageJ (National Institutes of Health, Bethesda, MD, USA).

Citrate synthase activity Tissue ( $\sim 5 \mathrm{mg} \mathrm{ww})$ was freeze-dried, dissected and homogenised (Tissuelyser; Qiagen) in $0.3 \mathrm{~mol} / 1$ phosphate buffer ( $\mathrm{pH} 7.7$ ) containing $0.05 \%$ BSA ( $3 \mathrm{~min}, 30$ revolutions/s). Maximal citrate synthase (CS) activity was determined, as previously described [34], using spectrophotometric readings at $405 \mathrm{~nm}$ absorbance (Multiscan; Thermo Fisher Scientific) at baseline and after the addition of oxaloacetate. CS activity was normalised to protein content.

\section{Calculations}

Insulin secretion rates (ISR) were deconvoluted from C-peptide measurements [35]. ISR as well as insulin levels were normalised to present glucose concentrations and analysed as absolute values as well as incremental to steadystate values of the previous clamp stage.

An insulin sensitivity index $\left[\mathrm{S}_{\mathrm{I}}=\mathrm{GIR} /(\right.$ insulin $\times$ glucose $\left.)\right]$ [36] was assessed during the last $30 \mathrm{~min}$ of the hyperglycaemic stage. DI was assessed in the hyperglycaemic stage as the late-phase (ISR/glucose) $\times \mathrm{S}_{\mathrm{I}}$.

\section{Statistical analyses}

Baseline variables were compared using one-way ANOVA. Potential within-group differences in variables of interest were assessed using two-way (group $\times$ time) repeated measures ANOVA, whereas between-group differences of the delta values (post-intervention minus pre-intervention) were assessed using one-way ANOVA. If a significant interaction arose, Bonferroni-corrected post-hoc tests were performed. Body compositional variables (body weight, fat mass and visceral fat content) were used as covariates in ANCOVA. Correlation analyses were performed to evaluate relationships between variables. All statistical analyses were performed using Prism v6 (GraphPad, San Diego, CA, USA) and Stata v13.1 (StataCorp LP, College Station, TX, USA). Statistical significance was accepted with $p<0.05$.

\section{Results}

Intervention and clinical variables (Tables 1 and 2)

No baseline differences were found between the groups for any of the following variables: sex, medication, age, physical activity, time since diagnosis, body composition, lipids or glycaemic control (fasting glucose and insulin, $\mathrm{HbA}_{1 \mathrm{c}}$, GIR during clamp stages, insulin secretion, $\left.\mathrm{S}_{\mathrm{I}}, R_{\mathrm{a}}, R_{\mathrm{d}}, \mathrm{DI}\right)$. As previously reported, mean training adherence was $89 \pm 4 \%$, with no differences between the IWT and CWT groups [6]. Both training groups were more physically active during the intervention compared with the $\mathrm{CON}$ group, whereas training amount, training-derived energy expenditure and mean training intensity did not differ between the IWT and CWT groups [6]. Despite that, IWT increased both absolute and relative maximal energy expenditure rate $\left(\dot{V} \mathrm{O}_{2 \max }\right)$ and induced weight and fat mass (total and visceral) loss, whereas no changes were seen in the CWT or CON groups [6].

GIR, insulin sensitivity and DI (Fig. 1, Table 2)

IWT increased GIR in the hyperglycaemic stage by $34.1 \pm$ $12.6 \%(p<0.05)$ and in the hyperglycaemic + GLP-1 stage by $34.4 \pm 10.7 \%(p<0.01)$. IWT increased $\mathrm{S}_{\mathrm{I}}$ and DI in the 
Table 1 Baseline characteristics and clinical and intervention variables

\begin{tabular}{|c|c|c|c|c|c|c|}
\hline \multirow[t]{2}{*}{ Characteristic/variable } & \multicolumn{2}{|l|}{$\mathrm{CON}$} & \multicolumn{2}{|l|}{ CWT } & \multicolumn{2}{|l|}{ IWT } \\
\hline & Pre- & Post- & Pre- & Post- & Pre- & Post- \\
\hline$n$ & 8 & & 12 & & 12 & \\
\hline Sex, $M / F$ & $5 / 3$ & & $8 / 4$ & & $7 / 5$ & \\
\hline Age, years & $57.1 \pm 3.0$ & & $60.8 \pm 2.2$ & & $57.5 \pm 2.4$ & \\
\hline Time since diagnosis, years & $4.5 \pm 1.5$ & & $6.2 \pm 1.5$ & & $3.5 \pm 0.7$ & \\
\hline \multicolumn{7}{|l|}{ Medication } \\
\hline Diet only & 5 & & 4 & & 4 & \\
\hline Metformin & 3 & & 7 & & 7 & \\
\hline Sulfonylureas & 1 & & 3 & & 3 & \\
\hline DPP-4 inhibitors & 1 & & 0 & & 1 & \\
\hline GLP-1 analogues & 1 & & 1 & & 1 & \\
\hline \multicolumn{7}{|l|}{$\dot{V} \mathrm{O}_{2 \max }$} \\
\hline Relative, $\mathrm{ml} \mathrm{kg}^{-1} \min ^{-1 \neq \uparrow}$ & $24.8 \pm 1.8$ & $25.2 \pm 2.0$ & $26.1 \pm 1.4$ & $26.8 \pm 1.9$ & $27.1 \pm 1.5$ & $31.5 \pm 2.2^{* * * \dagger \dagger}$ \\
\hline Absolute, $\mathrm{ml} / \mathrm{min}^{\neq \bullet}$ & $2,197 \pm 189$ & $2,228 \pm 181$ & $2,271 \pm 118$ & $2,281 \pm 126$ & $2,275 \pm 156$ & $2,524 \pm 204^{* *}$ \\
\hline \multicolumn{7}{|l|}{ Body composition } \\
\hline Body mass, $\mathrm{kg}^{\neq \|}$ & $88.5 \pm 4.7$ & $89.2 \pm 5.2$ & $88.2 \pm 4.7$ & $87.5 \pm 4.8$ & $84.9 \pm 4.9$ & $80.7 \pm 4.1^{* * *+\dagger t}$ \\
\hline Fat mass, $\mathrm{kg}^{\neq \boldsymbol{*}}$ & $28.2 \pm 3.2$ & $28.7 \pm 2.9$ & $28.3 \pm 3.1$ & $28.2 \pm 3.2$ & $28.8 \pm 2.7$ & $25.7 \pm 2.7^{* * *+\dagger+t}$ \\
\hline Visceral fat, $1^{\neq \boldsymbol{\oplus}}$ & $4.7 \pm 0.4$ & $4.6 \pm 0.4$ & $4.5 \pm 0.3$ & $4.2 \pm 0.4$ & $4.7 \pm 0.8$ & $4.2 \pm 0.7^{* * *}$ \\
\hline \multicolumn{7}{|l|}{ Lipids } \\
\hline Total cholesterol, mmol// ${ }^{\mp \oplus}$ & $5.4 \pm 0.2$ & $5.9 \pm 0.4^{*}$ & $5.3 \pm 0.3$ & $5.5 \pm 0.3$ & $5.0 \pm 0.3$ & $4.9 \pm 0.2^{\dagger}$ \\
\hline HDL-cholesterol, mmol/1 & $1.4 \pm 0.1$ & $1.4 \pm 0.2$ & $1.4 \pm 0.1$ & $1.4 \pm 0.1$ & $1.1 \pm 0.1$ & $1.1 \pm 0.1$ \\
\hline LDL-cholesterol, $\mathrm{mmol} / \mathrm{I}^{\top}$ & $3.4 \pm 0.1$ & $3.7 \pm 0.3$ & $3.3 \pm 0.2$ & $3.4 \pm 0.1$ & $3.2 \pm 0.2$ & $2.8 \pm 0.2^{* \dagger}$ \\
\hline Triacylglycerol, mmol/1 & $1.6 \pm 0.2$ & $1.9 \pm 0.3$ & $1.3 \pm 0.2$ & $1.5 \pm 0.3$ & $3.0 \pm 1.4$ & $2.5 \pm 0.8$ \\
\hline \multicolumn{7}{|l|}{ Intervention variables } \\
\hline Mean number of steps per day & & $8,144 \pm 1,169$ & & $11,780 \pm 693^{\S}$ & & $11,451 \pm 757^{\dagger}$ \\
\hline Training adherence, $\%$ of prescribed & & & & $94 \pm 6$ & & $85 \pm 4$ \\
\hline Total training-derived energy expenditure, MJ & & & & $117.8 \pm 13.5$ & & $121.4 \pm 12.5$ \\
\hline Mean training intensity (JD Mate), $\%^{\mathrm{a}}$ & & & & $72.7 \pm 3.6$ & & $70.5 \pm 2.0$ \\
\hline Mean training intensity (heart rate), $\%{ }^{\mathrm{b}}$ & & & & $66.4 \pm 1.4$ & & $65.7 \pm 1.7$ \\
\hline
\end{tabular}

Data are presented as mean \pm SEM

Some of the data included in this table have previously been published [6] and are used with the permission of the American Diabetes Association

${ }^{a}$ Fraction of mean training energy expenditure rates compared with the mean $\dot{V} \mathrm{O}_{2 \text { peak }}$ measured by the JD Mate

${ }^{\mathrm{b}}$ Fraction of mean training heart rates compared with the maximum heart rate measured during $\dot{V} \mathrm{O}_{2 \max }$ tests

Statistical differences are indicated by $\neq$ (main effect of time [by two-way repeated measures ANOVA across the groups]), 9 (time $\times$ group interaction [by two-way repeated measures ANOVA]), * (within group, pre- vs post-intervention), $\dagger(\Delta \mathrm{IWT}$ vs $\Delta \mathrm{CON}), \ddagger(\Delta \mathrm{IWT}$ vs $\Delta \mathrm{CWT}), \S(\mathrm{CON}$ vs CWT). No main effect of group (by two-way repeated measures ANOVA) was seen in any of the analyses. One symbol indicates $p<0.05$, two symbols indicate $p<0.01$ and three symbols indicate $p<0.001$

Pre-, before intervention; Post-, after a 4 month intervention; DPP-4, dipeptidyl peptidase-4

hyperglycaemic stage by $49.8 \pm 14.6 \%$ and $66.2 \pm 21.8 \%$, respectively ( $p<0.001$ for both). $R_{\mathrm{a}}$ was not altered by training in any of the stages, whereas peripheral glucose disposal $\left(R_{\mathrm{d}}\right)$ increased in the IWT group in the hyperglycaemic $(14.5 \pm 4.9 \%$; $p<0.05)$ and hyperglycaemic + GLP-1 stages $(25.0 \pm 9.9 \%$; $p<0.05)$. No intervention-induced changes were seen in either the CON or CWT groups for any of the variables.

ANCOVA analyses, where body composition variables were used as covariates, reduced the effect sizes on the above-mentioned variables in the IWT group by up to $25 \%$ (Table 3).

Insulin secretion (Figs 1 and 2)

Insulin secretion increased progressively through the clamp stages in all individuals. Besides increased fasting insulin within the CON group $(p<0.05)$, no significant changes 
Table 2 Hyperglycaemic clamp variables

\begin{tabular}{|c|c|c|c|c|c|c|}
\hline \multirow[t]{2}{*}{ Variable } & \multicolumn{2}{|l|}{$\mathrm{CON}$} & \multicolumn{2}{|l|}{ CWT } & \multicolumn{2}{|l|}{ IWT } \\
\hline & Pre- & Post- & Pre- & Post- & Pre- & Post- \\
\hline \multicolumn{7}{|l|}{ Basal } \\
\hline Glucose, mmol/1 & $7.6 \pm 0.9$ & $7.9 \pm 0.9$ & $7.7 \pm 0.6$ & $7.9 \pm 0.7$ & $9.0 \pm 0.9$ & $8.7 \pm 1.1$ \\
\hline Insulin, $\mathrm{pmol} / \mathrm{I}^{\top}$ & $88.8 \pm 9.8$ & $117.1 \pm 15.6^{*}$ & $93.6 \pm 13.4$ & $90.7 \pm 13.7$ & $96.2 \pm 13.3$ & $76.8 \pm 8.2^{\dagger}$ \\
\hline C-peptide, pmol/1 & $973 \pm 71$ & $1,113 \pm 110$ & $1,039 \pm 64$ & $1,019 \pm 80$ & $1,115 \pm 93$ & $977 \pm 55$ \\
\hline Glucagon, pmol/1 & $19.7 \pm 1.9$ & $21.1 \pm 3.2$ & $18.2 \pm 2.1$ & $18.8 \pm 3.1$ & $19.3 \pm 3.3$ & $20.7 \pm 4.3$ \\
\hline GLP-1, pmol/1 & $14.8 \pm 3.5$ & $17.5 \pm 1.7$ & $12.3 \pm 1.4$ & $12.0 \pm 1.4$ & $13.1 \pm 1.3$ & $15.3 \pm 1.4$ \\
\hline$R_{\mathrm{a}}, \mathrm{mg} \mathrm{kg}^{-1} \min ^{-1}$ & $2.1 \pm 0.2$ & $2.2 \pm 0.3$ & $2.5 \pm 0.3$ & $2.3 \pm 0.1$ & $2.1 \pm 0.2$ & $2.3 \pm 0.2$ \\
\hline \multicolumn{7}{|l|}{ Hyperglycaemic stage } \\
\hline Glucose, mmol/1 & $12.9 \pm 0.9$ & $12.8 \pm 0.9$ & $12.9 \pm 0.7$ & $12.9 \pm 0.6$ & $14.2 \pm 1.0$ & $14.1 \pm 1.0$ \\
\hline $\mathrm{CV}, \%$ & $1.8 \pm 0.3$ & $1.4 \pm 0.2$ & $1.3 \pm 0.3$ & $1.0 \pm 0.2$ & $1.2 \pm 0.3$ & $1.2 \pm 0.2$ \\
\hline Error from clamp goal, \% & $-1.0 \pm 0.3$ & $-1.7 \pm 0.9$ & $-1.3 \pm 0.5$ & $-1.7 \pm 0.5$ & $-1.6 \pm 0.6$ & $-1.9 \pm 0.5$ \\
\hline Insulin, pmol/1 & $326 \pm 81$ & $353 \pm 83$ & $363 \pm 102$ & $345 \pm 77$ & $235 \pm 42$ & $329 \pm 68$ \\
\hline C-peptide, pmol/1 & $2,261 \pm 335$ & $2,388 \pm 382$ & $2,398 \pm 297$ & $2,210 \pm 236$ & $2,106 \pm 225$ & $2,113 \pm 257$ \\
\hline Glucagon, pmol/1 & $13.6 \pm 3.1$ & $15.3 \pm 3.6$ & $9.5 \pm 1.9$ & $11.1 \pm 2.9$ & $13.5 \pm 2.6$ & $14.5 \pm 4.0$ \\
\hline GLP-1, pmol/1 & $15.5 \pm 3.3$ & $17.3 \pm 1.7$ & $14.1 \pm 1.7$ & $12.9 \pm 1.7$ & $13.6 \pm 1.1$ & $14.8 \pm 1.5$ \\
\hline GIR, $\mathrm{mg} \mathrm{kg}^{-1} \min ^{-1 \uparrow}$ & $2.6 \pm 0.3$ & $2.4 \pm 0.5$ & $2.6 \pm 0.3$ & $2.6 \pm 0.3$ & $2.4 \pm 0.3$ & $3.2 \pm 0.5^{*}$ \\
\hline$R_{\mathrm{a}}, \mathrm{mg} \mathrm{kg}^{-1} \min ^{-1}$ & $2.0 \pm 0.4$ & $1.9 \pm 0.5$ & $2.4 \pm 0.5$ & $2.0 \pm 0.3$ & $2.2 \pm 0.2$ & $2.2 \pm 0.5$ \\
\hline$R_{\mathrm{d}}, \mathrm{mg} \mathrm{kg}^{-1} \min ^{-1 \Phi}$ & $4.5 \pm 0.2$ & $4.4 \pm 0.3$ & $5.2 \pm 0.3$ & $4.8 \pm 0.3$ & $4.7 \pm 0.3$ & $5.4 \pm 0.3^{*+*}$ \\
\hline \multicolumn{7}{|l|}{ Hyperglycaemic+GLP-1 stage } \\
\hline Glucose, $\mathrm{mmol} / \mathrm{l}$ & $12.5 \pm 0.9$ & $12.6 \pm 0.9$ & $12.6 \pm 0.7$ & $12.7 \pm 0.7$ & $14.0 \pm 1.0$ & $13.8 \pm 1.0$ \\
\hline $\mathrm{CV}, \%$ & $3.3 \pm 0.8$ & $2.6 \pm 0.7$ & $2.9 \pm 0.7$ & $3.3 \pm 0.8$ & $2.3 \pm 0.4$ & $3.4 \pm 0.6$ \\
\hline Error from clamp goal, \% & $-3.9 \pm 0.7$ & $-3.0 \pm 0.8$ & $-3.6 \pm 0.7$ & $-3.3 \pm 0.6$ & $-3.0 \pm 0.5$ & $-4.2 \pm 0.6$ \\
\hline Insulin, pmol/1 & $1,364 \pm 449$ & $1,490 \pm 526$ & $1,676 \pm 548$ & $1,455 \pm 447$ & $783 \pm 149$ & $1,011 \pm 239$ \\
\hline C-peptide, pmol/1 & $4,708 \pm 799$ & $4,879 \pm 966$ & $5,287 \pm 762$ & $5,181 \pm 798$ & $4,357 \pm 472$ & $4,931 \pm 683$ \\
\hline Glucagon, pmol/1 & $7.2 \pm 2.8$ & $10.7 \pm 2.8$ & $5.9 \pm 8.4$ & $6.9 \pm 2.4$ & $8.4 \pm 2.1$ & $11.4 \pm 3.6$ \\
\hline GLP-1, pmol/1 & $63.4 \pm 4.6$ & $60.0 \pm 5.2$ & $58.4 \pm 2.2$ & $60.5 \pm 1.9$ & $59.0 \pm 2.8$ & $62.8 \pm 2.0$ \\
\hline GIR, $\mathrm{mg} \mathrm{kg}^{-1} \min ^{-1 \uparrow}$ & $6.4 \pm 1.2$ & $6.4 \pm 1.3$ & $7.0 \pm 1.0$ & $7.1 \pm 1.2$ & $6.1 \pm 0.8$ & $9.3 \pm 1.3^{* *}$ \\
\hline$R_{\mathrm{a}}, \mathrm{mg} \mathrm{kg}^{-1} \min ^{-1}$ & $0.3 \pm 0.2$ & $0.6 \pm 0.3$ & $0.7 \pm 0.3$ & $0.3 \pm 0.2$ & $0.4 \pm 0.2$ & $0.3 \pm 0.3$ \\
\hline$R_{\mathrm{d}}, \mathrm{mg} \mathrm{kg}^{-1} \min ^{-1 \uparrow}$ & $5.5 \pm 0.5$ & $5.4 \pm 0.5$ & $6.2 \pm 0.4$ & $5.8 \pm 0.5$ & $5.6 \pm 0.4$ & $7.0 \pm 0.7^{*}$ \\
\hline \multicolumn{7}{|l|}{ Arginine stage } \\
\hline Glucose, $\mathrm{mmol} / \mathrm{l}$ & $12.7 \pm 0.9$ & $12.8 \pm 0.8$ & $12.9 \pm 0.7$ & $12.4 \pm 0.5$ & $14.1 \pm 0.9$ & $14.2 \pm 0.9$ \\
\hline $\mathrm{CV}, \%$ & $4.6 \pm 0.8$ & $2.8 \pm 0.6$ & $2.8 \pm 0.4$ & $3.2 \pm 0.5$ & $3.0 \pm 0.7$ & $2.9 \pm 0.4$ \\
\hline Error from clamp goal, $\%$ & $-2.0 \pm 1.0$ & $-1.1 \pm 0.4$ & $-1.2 \pm 0.5$ & $-2.5 \pm 1.0$ & $-2.1 \pm 0.6$ & $-1.5 \pm 0.6$ \\
\hline Insulin, pmol/1 & $2,892 \pm 771$ & $2,629 \pm 809$ & $3,001 \pm 884$ & $2,884 \pm 868$ & $2,346 \pm 356$ & $2,491 \pm 435$ \\
\hline Maximal insulin, pmol/1 & $3,693 \pm 913$ & $3,416 \pm 1,003$ & $3,768 \pm 1,031$ & $3,631 \pm 1,024$ & $3,050 \pm 480$ & $3,172 \pm 560$ \\
\hline C-peptide, $\mathrm{pmol} / \mathrm{l}$ & $7,157 \pm 1,296$ & $6,762 \pm 1,384$ & $7,636 \pm 1,151$ & $7,525 \pm 1,164$ & $7,227 \pm 682$ & $7,767 \pm 931$ \\
\hline
\end{tabular}

Data are presented as mean $\pm \mathrm{SEM}$ during the last 30 min of the hyperglycaemic and hyperglycaemic + GLP-1 stages and mean \pm SEM during the first $6 \mathrm{~min}$ of the arginine stage

Statistical differences are indicated by 9 (time $\times$ group interaction [by two-way repeated measures ANOVA]), * (within group, pre- vs post-intervention), $\dagger(\triangle \mathrm{IWT}$ vs $\Delta \mathrm{CON}), \ddagger(\Delta \mathrm{IWT}$ vs $\Delta \mathrm{CWT})$. No main effects of group or time [by two-way repeated measures ANOVA] were seen in any of the analyses. One symbol indicates $p<0.05$, two symbols indicate $p<0.01$ and three symbols indicate $p<0.001$

Pre-, before intervention; Post-, after a 4 month intervention

within groups or differences between groups in insulin levels or ISR were found either in the OGTT or in the hyperglycaemic clamp (any stage; acute phase and late phase).

\section{Glucagon}

Glucagon values in all individuals declined progressively from fasting levels $(19.5 \pm 1.3 \mathrm{pmol} / \mathrm{l})$ to hyperglycaemia 


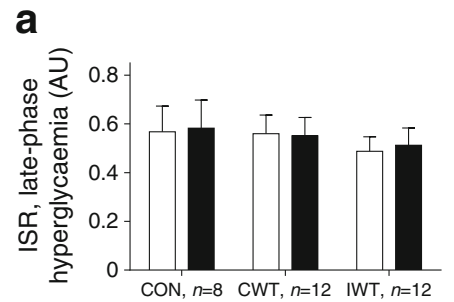

b

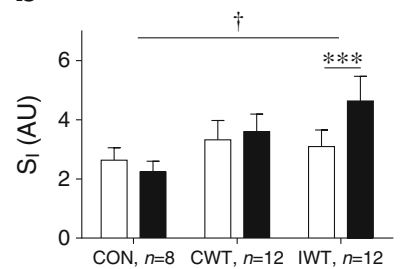

C

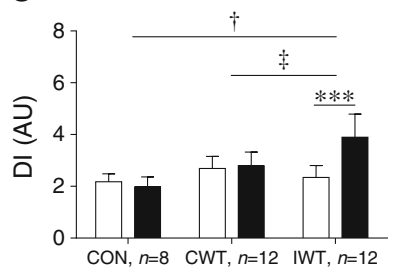

Fig. 1 ISR normalised to glucose concentrations measured during the late hyperglycaemic clamp stage (last $30 \mathrm{~min})(\mathbf{a}), \mathrm{S}_{\mathrm{I}}(\mathbf{b})$, DI (c). White bars, pre-intervention; black bars, post-intervention. Data are presented as

$(12.7 \pm 1.2 \mathrm{pmol} / 1 ; p<0.001)$ to hyperglycaemia + GLP-1 ( $8.4 \pm 1.1 \mathrm{pmol} / 1 ; p<0.001$ vs both fasting and hyperglycaemia), mean \pm SEM. Statistical differences indicated by $* * * p<0.001$ within groups and by ${ }^{\dagger} p<0.05$ (CON vs IWT) and ${ }^{\ddagger} p<0.05$ (CWT vs IWT) between groups

with no significant changes within groups or differences between groups.

Table 3 Impact of body compositional changes

\begin{tabular}{|c|c|c|c|c|}
\hline Variable & $\Delta \mathrm{CON}$ & $\Delta \mathrm{CWT}$ & $\Delta \mathrm{IWT}$ & $\Delta$ IWT covariate $-\Delta$ IWT crude \\
\hline \multicolumn{5}{|l|}{ GIR, hyperglycaemic stage } \\
\hline Unadjusted & $-0.14 \pm 0.28$ & $-0.05 \pm 0.23$ & $0.81 \pm 0.23^{*}$ & \\
\hline Body weight-adjusted & $-0.03 \pm 0.27$ & $-0.07 \pm 0.22$ & $0.73 \pm 0.23^{*}$ & 0.91 \\
\hline Fat mass-adjusted & $0.07 \pm 0.30$ & $-0.05 \pm 0.23$ & $0.79 \pm 0.23^{*}$ & 0.98 \\
\hline Visceral fat content-adjusted & $-0.05 \pm 0.28$ & $-0.18 \pm 0.25$ & $0.76 \pm 0.24^{*}$ & 0.94 \\
\hline \multicolumn{5}{|c|}{ GIR, hyperglycaemic + GLP-1 stage } \\
\hline Unadjusted & $-0.05 \pm 0.97$ & $0.16 \pm 0.80$ & $3.21 \pm 0.80^{* *}$ & \\
\hline Body weight-adjusted & $-0.04 \pm 0.96$ & $0.15 \pm 0.79$ & $3.12 \pm 0.80^{* *}$ & 0.97 \\
\hline Fat mass-adjusted & $-0.05 \pm 1.07$ & $0.17 \pm 0.82$ & $3.37 \pm 0.84^{* *}$ & 1.05 \\
\hline Visceral fat content-adjusted & $-0.05 \pm 0.84$ & $0.82 \pm 0.76$ & $3.21 \pm 0.71^{* *}$ & 1.00 \\
\hline \multicolumn{5}{|l|}{$\mathrm{S}_{\mathrm{I}}$} \\
\hline Unadjusted & $-0.46 \pm 0.70$ & $0.35 \pm 0.57$ & $2.66 \pm 0.57^{* * *}$ & \\
\hline Body weight-adjusted & $-0.55 \pm 0.73$ & $0.34 \pm 0.59$ & $1.92 \pm 0.61^{*}$ & 0.75 \\
\hline Fat mass-adjusted & $-0.55 \pm 0.76$ & $0.44 \pm 0.58$ & $2.21 \pm 0.61^{* *}$ & 0.86 \\
\hline Visceral fat content-adjusted & $-0.76 \pm 0.77$ & $-0.53 \pm 0.69$ & $1.94 \pm 0.64^{*}$ & 0.76 \\
\hline \multicolumn{5}{|l|}{ DI } \\
\hline Unadjusted & $-0.18 \pm 0.42$ & $0.29 \pm 0.34$ & $1.57 \pm 0.34^{* * *}$ & \\
\hline Body weight-adjusted & $-0.13 \pm 0.42$ & $0.25 \pm 0.3$ & $1.34 \pm 0.35^{* *}$ & 0.86 \\
\hline Fat mass-adjusted & $-0.11 \pm 0.45$ & $0.29 \pm 0.34$ & $1.45 \pm 0.36^{* *}$ & 0.93 \\
\hline Visceral fat content-adjusted & $-0.21 \pm 0.45$ & $0.19 \pm 0.40$ & $1.36 \pm 0.38^{* *}$ & 0.87 \\
\hline \multicolumn{5}{|l|}{$R_{\mathrm{d}}$, hyperglycaemic stage } \\
\hline Unadjusted & $-0.10 \pm 0.2$ & $-0.39 \pm 0.21$ & $0.68 \pm 0.21^{*}$ & \\
\hline Body weight-adjusted & $-0.08 \pm 0.25$ & $-0.40 \pm 0.21$ & $0.60 \pm 0.21^{*}$ & 0.88 \\
\hline Fat mass-adjusted & $-0.12 \pm 0.28$ & $-0.39 \pm 0.21$ & $0.54 \pm 0.22$ & 0.80 \\
\hline Visceral fat content-adjusted & $-0.10 \pm 0.22$ & $-0.25 \pm 0.20$ & $0.64 \pm 0.18^{*}$ & 0.94 \\
\hline \multicolumn{5}{|l|}{$R_{\mathrm{d}}$, hyperglycaemic + GLP-1 stage } \\
\hline Unadjusted & $-0.12 \pm 0.50$ & $-0.43 \pm 0.41$ & $1.41 \pm 0.41^{*}$ & \\
\hline Body weight-adjusted & $-0.11 \pm 0.50$ & $-0.44 \pm 0.4$ & $1.39 \pm 0.41^{*}$ & 0.98 \\
\hline Fat mass-adjusted & $-0.15 \pm 0.55$ & $-0.43 \pm 0.42$ & $1.43 \pm 0.43^{*}$ & 1.01 \\
\hline Visceral fat content-adjusted & $-0.11 \pm 0.50$ & $-0.25 \pm 0.45$ & $1.47 \pm 0.42^{*}$ & 1.04 \\
\hline
\end{tabular}

Data in the first three rows are presented as mean \pm SEM of the delta values (within-group differences between pre- and post-intervention values)

Data in the last row are the ratio between the covariate-adjusted delta value and the unadjusted delta value in the IWT group. Thus, a value of 0.91 indicates that $91 \%$ of the absolute difference between pre- and post-intervention values remained after adjusting for the covariate

Bonferroni-corrected statistical differences are indicated by * (within group, pre- vs post-intervention). One symbol indicates $p<0.05$, two symbols indicate $p<0.01$ and three symbols indicate $p<0.001$ 


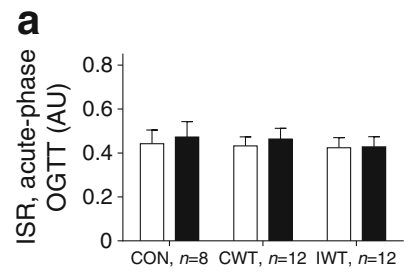

b


e

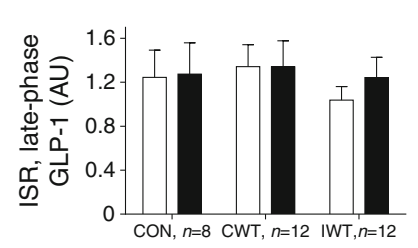

C

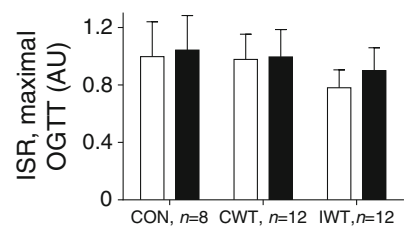

f

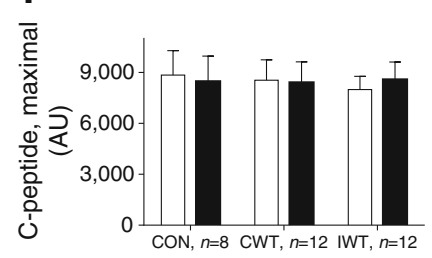

Fig. 2 ISR normalised to glucose concentrations measured during (a) OGTT (acute phase; 0-20 min), (b) OGTT (total secretion; 0-180 min), (c) OGTT (maximal secretion), (d) clamp (acute hyperglycaemic stage; 0-6 min), (e) clamp (late hyperglycaemic + GLP-1 stage; last $30 \mathrm{~min}$ ). (f)
Maximal C-peptide during the arginine stage. White bars, pre-intervention; black bars, post-intervention. Data are presented as mean $\pm \mathrm{SEM}$. No significant differences were found
Insulin signalling (Fig. 3)

GLUT4 mRNA levels did not change in the CON or CWT groups, whereas increases were seen in the IWT group $(41.7 \pm$
$11.5 \% ; p<0.01)$; however, this was not reflected by increased GLUT4 protein. The pAkt $\left({ }^{473} \mathrm{Ser}\right) / \mathrm{Akt}$ ratio increased with hyperglycaemia in all groups $(p<0.05)$, but no interventioninduced changes within groups or differences between groups

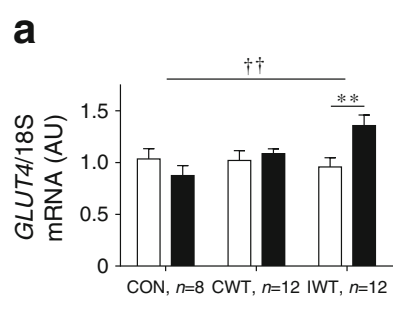

b

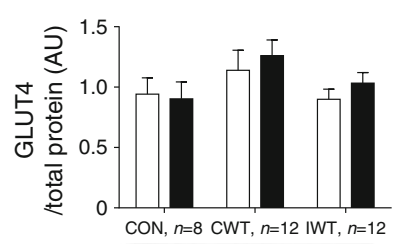

$55 \mathrm{kDa} \rightarrow$



C

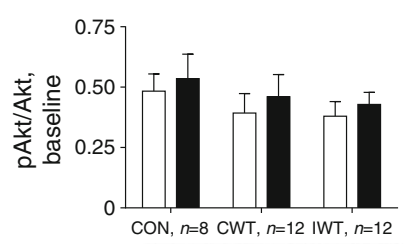

$60 \mathrm{kDa}(\mathrm{pAkt}) \rightarrow$

$60 \mathrm{kDa}($ Akt) $\rightarrow-m-\cdots$
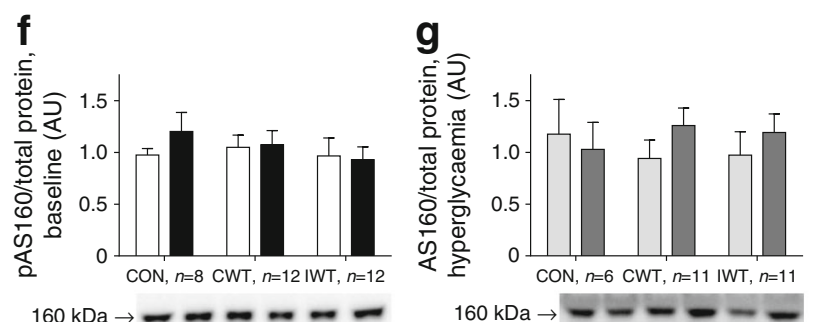

$160 \mathrm{kDa} \rightarrow-----$ d

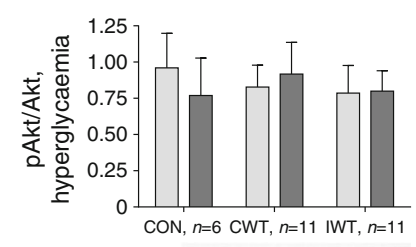

$60 \mathrm{kDa}$ (pAkt) $\rightarrow-m=-m=$

$60 \mathrm{kDa}($ Akt) $\rightarrow-m-m$

h



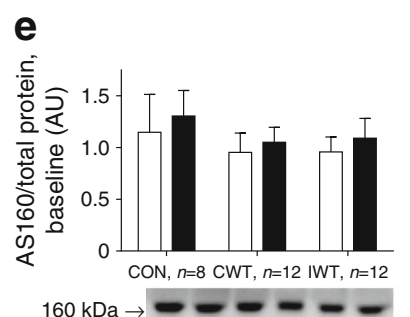

$160 \mathrm{kDa} \rightarrow--\infty-\infty$

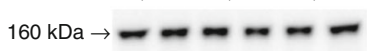

$160 \mathrm{kDa} \rightarrow-\cdots \cdots$

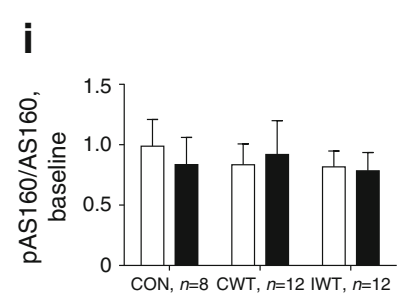

Fig. 3 Insulin signalling variables measured in skeletal muscle tissue at baseline: (a) GLUT4 mRNA, (b) GLUT4 protein, (c) pAkt $\left({ }^{473} \mathrm{Ser}\right) / \mathrm{Akt}$ protein, (e) AS160 protein, (f) pAS160( ${ }^{642} \mathrm{Thr}$, (i) pAS160 $\left({ }^{642} \mathrm{Thr}\right) /$ AS160); and during hyperglycaemia: (d) $\mathrm{pAkt}\left({ }^{473} \mathrm{Ser}\right) / \mathrm{Akt}$ protein, (g) AS160, (h) pAS160 $\left({ }^{642} \mathrm{Thr}\right)$, (j) pAS160 $\left({ }^{642} \mathrm{Thr}\right) / \mathrm{AS} 160$. (k) Loading

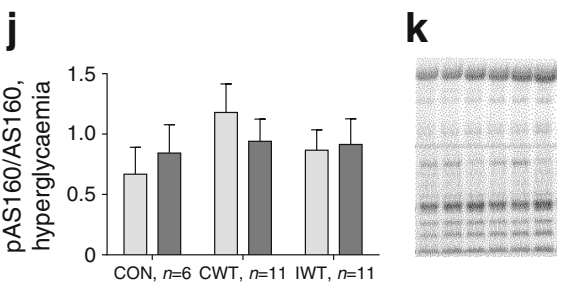

controls (Reactive Brown). White/light grey bars, pre-intervention; black/dark grey bars, post-intervention. Data are presented as mean \pm SEM. Statistical differences indicated by $* * p<0.01$ and $* p<0.05$ within groups and by ${ }^{\dagger \dagger} p<0.01$ between groups 
were found either during fasting or during glucose stimulation. Moreover, neither Akt nor pAkt $\left({ }^{473}\right.$ Ser $)$ alone changed with the intervention in any of the groups. pAS160 $\left({ }^{642} \mathrm{Thr}\right)$ increased with hyperglycaemia in response to the intervention in the IWT group (29.0土 $10.8 \% ; p<0.05)$. No significant changes were found for pAS160 $\left({ }^{642} \mathrm{Thr}\right)$ in the other groups, and no significant changes within groups or differences between groups were found for total AS160 protein. Moreover, no changes were found within or between groups for the pAS160 $\left({ }^{642} \mathrm{Thr}\right) / \mathrm{AS} 160$ ratio.

\section{Glycogen (Fig. 4)}

Glycogen phosphorylase mRNA increased in the IWT group $(39.4 \pm 11.7 \% ; p<0.05)$. No changes were seen in the CON or CWT groups. Delta values of glycogen synthase mRNA were higher in the IWT group compared with the CON group $(p<0.05)$, whereas protein levels of hexokinase 2 and glycogen synthase kinase (GSK)-3 $\beta$ did not differ between groups or change in any of the groups. No differences or changes were found in skeletal muscle glycogen content in any of the groups, either during baseline or during hyperglycaemia, and no significant differences between fasting and glucose-stimulated glycogen levels were found in any of the groups.
Mitochondrial markers (Fig. 5)

In the IWT group, $C S$ mRNA increased after the intervention $(28.1 \pm 8.3 \% ; p<0.01)$, whereas CS activity remained unchanged. No changes were seen in the CON or CWT groups. No changes were seen in peroxisome proliferatoractivated receptor gamma coactivator $1 \alpha(P G C-1 \alpha)$ mRNA, cytochrome $c$ or COX-4 protein levels in any of the groups. Correlation analyses showed no significant relationships between changes in mitochondrial markers and changes in GIR, $\mathrm{S}_{\mathrm{I}}, R_{\mathrm{a}}, R_{\mathrm{d}}$ or DI.

\section{Discussion}

The most important finding of this study is that IWT, but not CWT, increased $\mathrm{S}_{\mathrm{I}}$ without a compensatory decrease in insulin secretion, thus increasing the DI. This explains why interval, compared with continuous, training is favourable for improving glycaemic control in individuals with type 2 diabetes [6]. Another important observation is that continuous training was not advantageous compared with a non-training control group, with respect to $S_{I}$, insulin secretion and glucose disposition. A major strength of our design includes the successful matching of training amount, energy expenditure and mean training
Fig. 4 Glycogen variables measured in skeletal muscle tissue at baseline: (a) Glycogen synthase (GYS1) mRNA, (b) Glycogen phosphorylase $(P Y G)$ mRNA, (c) GSK-3 $\beta$ protein, (d) Hexokinase 2 (HK2) protein, (f) baseline glycogen content; and during hyperglycaemia: (g) hyperglycaemic glycogen content. (e) Loading controls (Reactive Brown). White/light grey bars, pre-intervention; black/dark grey bars, postintervention. Data are presented as mean \pm SEM. Statistical differences indicated by $* p<0.05$ within groups and by ${ }^{\dagger} p<0.05$ between groups a



b
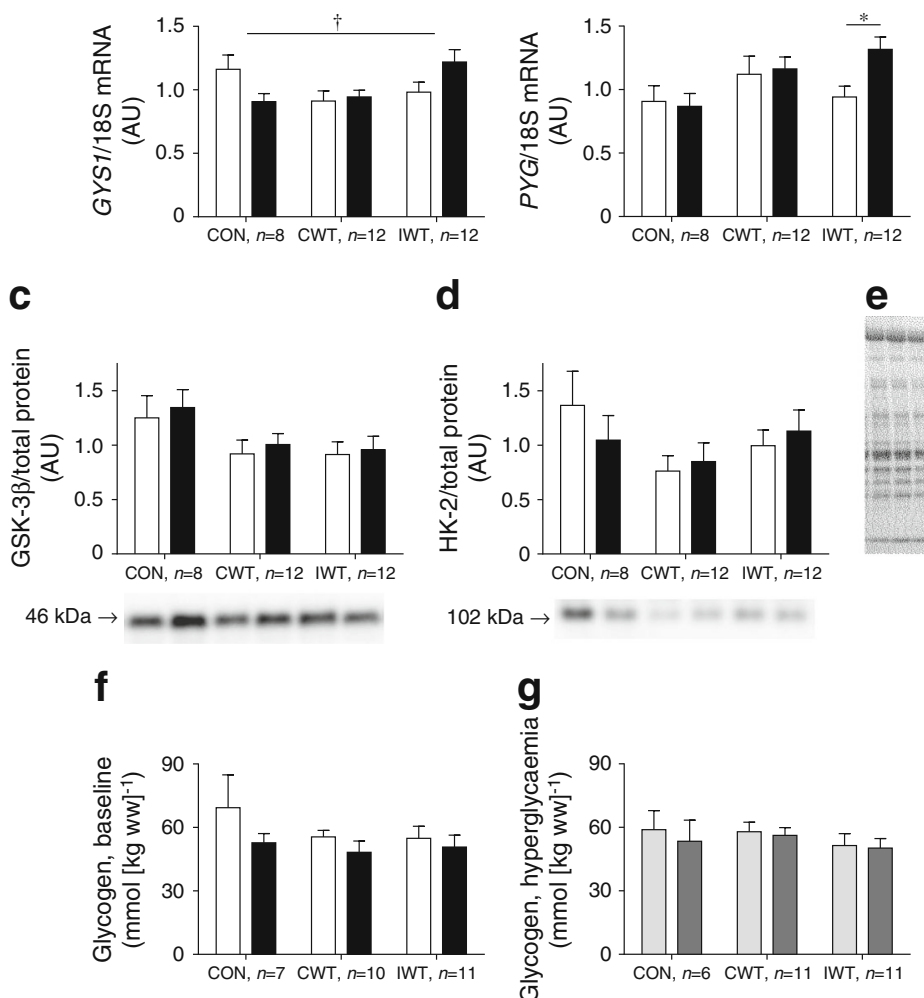
Fig. 5 Mitochondrial variables measured in skeletal muscle tissue at baseline: (a) $P G C-1 \alpha$ mRNA, (b) CS mRNA, (c) CS activity, (d) cytochrome $c$ (CYT C) protein, (e) COX-4 protein. (f) Loading controls (Reactive Brown). White bars, pre-intervention; black bars, post-intervention. Data are presented as mean $\pm \mathrm{SEM}$. Statistical differences indicated by ${ }^{* *} p<0.01$ and by ${ }^{\dagger} p<0.05$ between groups
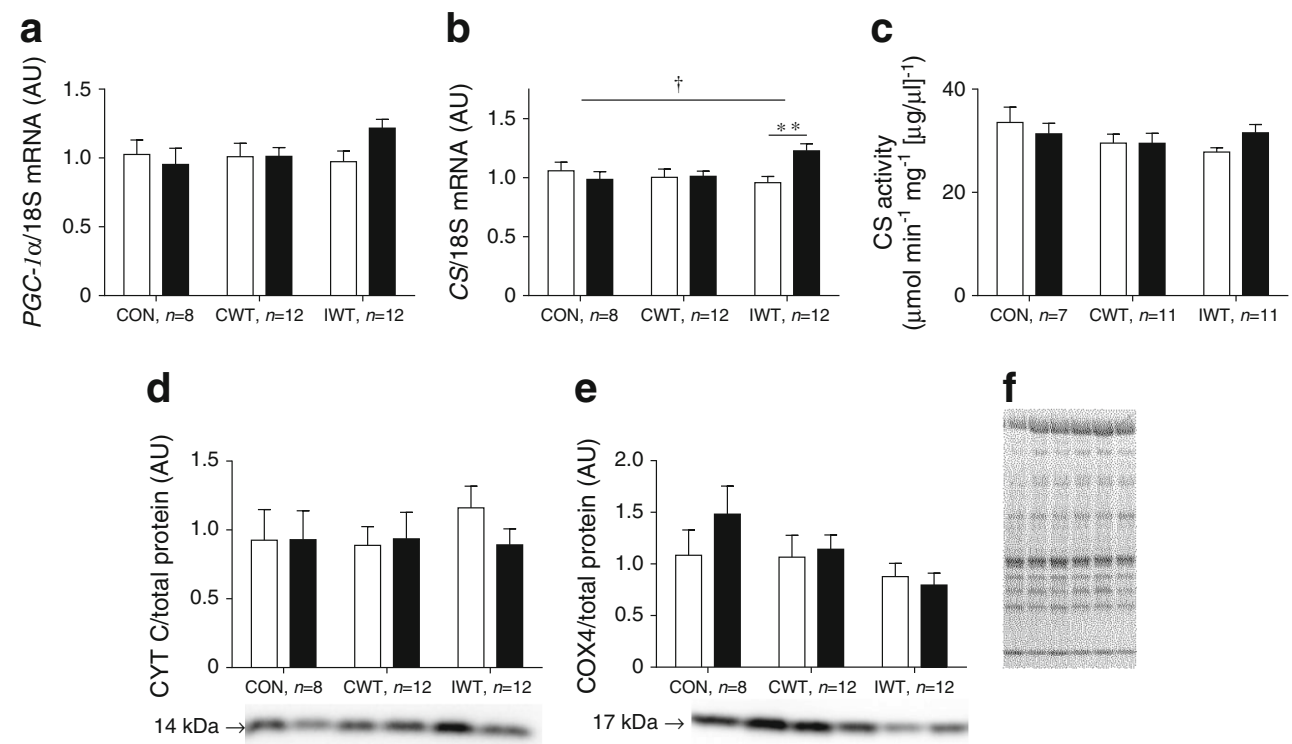

intensity between IWT and CWT, which justifies comparison between them.

The lack of changes in the CWT group is surprising and is in disagreement with other studies [10,12,13]. Individuals were instructed to train for $60 \mathrm{~min}$, five times weekly for 4 months. With $94 \pm 6 \%$ completion of this training schedule and no differences in baseline or end intervention physical activity levels between the IWT and CWT groups [6], the lack of effects cannot be ascribed to a lack of training. We believe that the high training volume alone was not enough to elicit changes [37], and that the spikes and/or variability in training intensity in the IWT group were necessary for the improvements seen in metabolic outcome $[4,6,38]$ in this group of individuals who at baseline were fairly physical active and fit [6] compared with other cohorts [11-13]. Since weight loss per se is known to improve $S_{I}$ and glucose disposal [39, 40], and since the timing of our post-intervention measurements was such that training-induced effects on $\mathrm{S}_{\mathrm{I}}$ potentially would be present only in the case of improved body composition [40], one might speculate that the improved body composition in the IWT group, but not in the CWT group, would fully explain the difference in metabolic outcome between these groups. ANCOVA analyses, however, indicated that changes in body weight explained maximally $25 \%$ of the improvements seen, indicating that other factors contributed. Still, the differential body compositional outcome between IWT and CWT remains an important factor that must be taken into account when interpreting our results.

The increased $\mathrm{S}_{\mathrm{I}}$ following IWT was entirely explained by increased peripheral glucose disposal, as endogenous glucose production was unchanged. Thus, we expected that muscle insulin signalling would be improved in the IWT group [9]. Indeed, IWT increased AS160 phosphorylation during hyperglycaemia, whereas upstream Akt phosphorylation was unchanged. Likewise, Vind et al found that AS160 phosphorylation increased following endurance training in the absence of increased Akt phosphorylation in individuals with type 2 diabetes [41]. To our knowledge, however, we are the first to evaluate the effect of continuous vs interval training on these variables. Surprisingly, GLUT4 protein was not significantly increased after IWT. Total GLUT4 protein level does not, however, necessarily reflect the amount of GLUT4 in the plasma membrane, which is the important pool to consider when glucose uptake is of interest [42]. Since phosphorylation of AS160 is known to increase docking of GLUT4-containing vesicles to the cell membrane in skeletal muscle cells [43], it is likely that more membrane-bound GLUT4 may explain the increase in glucose disposal in the IWT group in contrast to the CWT group. Also, although no significant changes in fasting glucose were seen after any intervention, individual changes may have influenced GIRs and therefore $\mathrm{S}_{\mathrm{I}}$. Finally, although hyperglycaemic clamp-measured $\mathrm{S}_{\mathrm{I}}$ agrees well with hyperinsulinaemic-euglycaemic clamp-measured insulin sensitivity [44], glucose effectiveness is included in the hyperglycaemic clamp-measured $S_{I}$ [27]. In this context, Nishida et al showed that endurance training increases glucose effectiveness, which is sustained for 1 week after training cessation [45]. Thus, we cannot rule out that the increased $\mathrm{S}_{\text {I }}$ seen in the IWT group was dependent on increased glucose effectiveness.

We found no changes in insulin secretion. Because pretraining insulin secretory capacity $[16,17,20]$ and degree of hyperglycaemia [46] may influence exercise adaptations in insulin secretion, the lack of changes may be due to the large heterogeneity within our cohort (maximal baseline insulin secretion $0.77-7.79 \mathrm{pmol} \mathrm{kg}^{-1} \mathrm{~min}^{-1}(\mathrm{mmol} / \mathrm{l})^{-1}$; baseline $2 \mathrm{~h}$ OGTT glucose $9.5-22.5 \mathrm{mmol} / \mathrm{l})$. Importantly, however, despite a marked increase in $\mathrm{S}_{\mathrm{I}}$, no signs of decreased insulin 
secretion were found following interval training, as typically observed in non-diabetic populations [22], and, as a result, IWT increased the DI in contrast to CWT and CON. Glucagon secretion was unchanged after the intervention, which is in accordance with the observed unaffected endogenous glucose production.

Impaired glycogen storage and turnover are involved in the development of type 2 diabetes [47], and endurance training has been described to improve these variables in individuals with type 2 diabetes [48]. While some indication of improved muscle glycogen turnover was evident in the IWT group (increased glycogen phosphorylase mRNA and difference in $\triangle \mathrm{IWT}-\triangle \mathrm{CON}$ glycogen synthase mRNA), no differences were seen in GSK-3 $\beta$ protein, hexokinase 2 protein or glycogen levels. During the hyperglycaemic clamp, inter-individual insulin levels were highly variable (fasting range 23-196 pmol/l; hyperglycaemic range 63-1,275 pmol/1). This variability in insulin levels in addition to the semi-quantitative nature of western blotting may have blurred potential differences within and between groups in the proteins measured.

Mitochondrial content and function have been linked to insulin sensitivity, and changes in mitochondrial markers have been seen along with improvements in glycaemic control and insulin sensitivity [49, 50]. Of particular interest, interval training programmes have resulted in concurrent large improvements in mitochondrial markers and glucose metabolism $[4,5]$. In this study, however, no significant changes in the protein level or in CS activity were seen. Moreover, since no relationships were found between changes in mitochondrial markers and glucose metabolism, this study does not support a link between mitochondria and glucose metabolism. It must be noted, however, that we did not measure intrinsic mitochondrial function.

We used the hyperglycaemic clamp for mechanistic assessments since this allows evaluation of insulin secretion in addition to $S_{I}[27]$. Although the method considered the gold standard for measuring $\mathrm{S}_{\mathrm{I}}$ is the hyperinsulinaemiceuglycaemic clamp, direct comparisons of the two methods in individuals with type 2 diabetes have resulted in comparable results [36]. Also, we believe that the hyperglycaemic clamp is more physiological than the hyperinsulinaemiceuglycaemic clamp, as it represents a situation that individuals with type 2 diabetes face in real life (hyperglycaemia with a need for appropriate insulin secretion), in contrast to that of the hyperinsulinaemic-euglycaemic clamp (euglycaemia with artificially high and steady insulin levels).

In summary, improved glycaemic control in individuals with type 2 diabetes following interval training is explained by increased $\mathrm{S}_{\mathrm{I}}$ and maintained insulin secretory function, resulting in increased DI. These adaptations are not observed following energy expenditure-matched continuous training. Whether these beneficial effects extrapolate to hard endpoints in the long term must be determined in order to justify the clinical utility of interval training for individuals with type 2 diabetes.

Acknowledgements We acknowledge the following individuals for their technical assistance: M. Ried-Larsen, R. Rovsing, H. Villumsen, T. Grøndahl, R. M. Dahl and T. B. Kryger (all from The Centre of Inflammation and Metabolism and The Centre for Physical Activity Research, Department of Infectious Diseases and CMRC, Rigshospitalet, Faculty of Health Sciences, University of Copenhagen, Denmark) and G. van Hall (Clinical Metabolomics Core Facility, Department of Infectious Diseases and CMRC, Rigshospitalet, Faculty of Health Sciences, University of Copenhagen, Denmark).

Funding This study was primarily funded by DD2 (The Danish Centre for Strategic Research in Type 2 Diabetes) supported by the Danish Agency for Science (grants 09-067009 and 09-075724 to BKP). The project partners are listed on the project website, www.DD2.nu. This study was further supported by grants from the AP Møller Fonden (KK) and Trygfonden (BKP). The Centre of Inflammation and Metabolism (CIM) is supported by a grant from the Danish National Research Foundation (DNRF55). The Centre for Physical Activity Research (CFAS) is supported by a grant from Trygfonden. CIM is part of the UNIK Project, Food, Fitness and Pharma for Health and Disease, supported by the Danish Ministry of Science, Technology and Innovation. The Copenhagen Muscle Research Centre is supported by a grant from the Capital Region of Denmark.

Duality of interest The authors declare that there is no duality of interest associated with this manuscript.

Contribution statement KK and TPJS designed the study, acquired, analysed and interpreted the data and wrote the manuscript. KW, SHK, NGJ, MMS, JO, JJH and BKP analysed and interpreted the data and reviewed/edited the manuscript. All authors approved the final version of the manuscript. TPJS is responsible for the integrity of the work as a whole.

\section{References}

1. Albright A, Franz M, Hornsby G et al (2000) American College of Sports Medicine position stand. Exercise and type 2 diabetes. Med Sci Sports Exerc 32:1345-1360

2. Boule NG, Kenny GP, Haddad E, Wells GA, Sigal RJ (2003) Metaanalysis of the effect of structured exercise training on cardiorespiratory fitness in Type 2 diabetes mellitus. Diabetologia 46:1071-1081

3. Earnest CP (2008) Exercise interval training: an improved stimulus for improving the physiology of pre-diabetes. Med Hypotheses 71: 752-761

4. Tjonna AE, Lee SJ, Rognmo O et al (2008) Aerobic interval training versus continuous moderate exercise as a treatment for the metabolic syndrome: a pilot study. Circulation 118:346-354

5. Little JP, Gillen JB, Percival M, et al (2011) Low-volume highintensity interval training reduces hyperglycemia and increases muscle mitochondrial capacity in patients with type 2 diabetes. J Appl Physiol

6. Karstoft K, Winding K, Knudsen SH et al (2013) The effects of freeliving interval-walking training on glycemic control, body composition, and physical fitness in type 2 diabetes patients. Diabetes Care $36: 228-236$

7. Kirwan JP, Solomon TP, Wojta DM, Staten MA, Holloszy JO (2009) Effects of 7 days of exercise training on insulin 
sensitivity and responsiveness in type 2 diabetes mellitus. Am J Physiol Endocrinol Metab 297:E151-E156

8. Christ-Roberts CY, Pratipanawatr T, Pratipanawatr W et al (2004) Exercise training increases glycogen synthase activity and GLUT4 expression but not insulin signaling in overweight nondiabetic and type 2 diabetic subjects. Metabolism 53:1233-1242

9. Wang Y, Simar D, Fiatarone Singh MA (2009) Adaptations to exercise training within skeletal muscle in adults with type 2 diabetes or impaired glucose tolerance: a systematic review. Diabetes Metab Res Rev 25:13-40

10. Houmard JA, Tanner CJ, Slentz CA, Duscha BD, McCartney JS, Kraus WE (2004) Effect of the volume and intensity of exercise training on insulin sensitivity. J Appl Physiol 96:101-106

11. Kang J, Robertson RJ, Hagberg JM et al (1996) Effect of exercise intensity on glucose and insulin metabolism in obese individuals and obese NIDDM patients. Diabetes Care 19:341-349

12. Dube JJ, Allison KF, Rousson V, Goodpaster BH, Amati F (2012) Exercise dose and insulin sensitivity: relevance for diabetes prevention. Med Sci Sports Exerc 44:793-799

13. Hansen D, Dendale P, Jonkers RA et al (2009) Continuous low- to moderate-intensity exercise training is as effective as moderate- to high-intensity exercise training at lowering blood $\mathrm{HbA}(1 \mathrm{c})$ in obese type 2 diabetes patients. Diabetologia 52:1789-1797

14. Da Silva AS, Pauli JR, Ropelle ER et al (2010) Exercise intensity, inflammatory signaling, and insulin resistance in obese rats. Med Sci Sports Exerc 42:2180-2188

15. Koshinaka K, Sano A, Howlett KF et al (2008) Effect of highintensity intermittent swimming on postexercise insulin sensitivity in rat epitrochlearis muscle. Metabolism 57:749-756

16. Dela F, von Linstow ME, Mikines KJ, Galbo H (2004) Physical training may enhance beta-cell function in type 2 diabetes. Am J Physiol Endocrinol Metab 287:E1024-E1031

17. Krotkiewski M, Lonnroth P, Mandroukas K et al (1985) The effects of physical training on insulin secretion and effectiveness and on glucose metabolism in obesity and type 2 (non-insulin-dependent) diabetes mellitus. Diabetologia 28:881-890

18. Rogers MA, Yamamoto C, King DS, Hagberg JM, Ehsani AA, Holloszy JO (1988) Improvement in glucose tolerance after $1 \mathrm{wk}$ of exercise in patients with mild NIDDM. Diabetes Care 11:613-618

19. Eriksen L, Dahl-Petersen I, Haugaard SB, Dela F (2007) Comparison of the effect of multiple short-duration with single long-duration exercise sessions on glucose homeostasis in type 2 diabetes mellitus. Diabetologia 50:2245-2253

20. Solomon TP, Malin SK, Karstoft K, Kashyap SR, Haus JM, Kirwan JP (2013) Pancreatic beta-cell function is a stronger predictor of changes in glycemic control after an aerobic exercise intervention than insulin sensitivity. J Clin Endocrinol Metab 98:4176-4186

21. Utzschneider KM, Prigeon RL, Carr DB et al (2006) Impact of differences in fasting glucose and glucose tolerance on the hyperbolic relationship between insulin sensitivity and insulin responses. Diabetes Care 29:356-362

22. Slentz CA, Tanner CJ, Bateman LA et al (2009) Effects of exercise training intensity on pancreatic beta-cell function. Diabetes Care 32: $1807-1811$

23. Ryden L, Grant PJ, Anker SD et al (2013) ESC guidelines on diabetes, pre-diabetes, and cardiovascular diseases developed in collaboration with the EASD: the Task Force on diabetes, pre-diabetes, and cardiovascular diseases of the European Society of Cardiology (ESC) and developed in collaboration with the European Association for the Study of Diabetes (EASD). Eur Heart J 34:3035-3087

24. Pedersen BK, Saltin B (2006) Evidence for prescribing exercise as therapy in chronic disease. Scand J Med Sci Sports 16(Suppl 1): 3-63

25. Yamazaki T, Gen-no H, Kamijo Y, Okazaki K, Masuki S, Nose H (2009) A new device to estimate $\mathrm{VO} 2$ during incline walking by accelerometry and barometry. Med Sci Sports Exerc 41:2213-2219
26. Iwashita S, Takeno Y, Okazaki K et al (2003) Triaxial accelerometry to evaluate walking efficiency in older subjects. Med Sci Sports Exerc $35: 1766-1772$

27. Elahi D (1996) In praise of the hyperglycemic clamp. A method for assessment of beta-cell sensitivity and insulin resistance. Diabetes Care 19:278-286

28. DeFronzo RA, Tobin JD, Andres R (1979) Glucose clamp technique: a method for quantifying insulin secretion and resistance. Am J Physiol 237:E214-E223

29. Orskov C, Wettergren A, Holst JJ (1996) Secretion of the incretin hormones glucagon-like peptide-1 and gastric inhibitory polypeptide correlates with insulin secretion in normal man throughout the day. Scand J Gastroenterol 31:665-670

30. Bergstrom J (1975) Percutaneous needle biopsy of skeletal muscle in physiological and clinical research. Scand J Clin Lab Invest 35:609616

31. Oehlke J, Brudel M, Blasig IE (1994) Benzoylation of sugars, polyols and amino acids in biological fluids for high-performance liquid chromatographic analysis. J Chromatogr B Biomed Appl 655:105111

32. Plomgaard P, Bouzakri K, Krogh-Madsen R, Mittendorfer B, Zierath JR, Pedersen BK (2005) Tumor necrosis factor-alpha induces skeletal muscle insulin resistance in healthy human subjects via inhibition of Akt substrate 160 phosphorylation. Diabetes 54:2939-2945

33. Orskov C, Rabenhoj L, Wettergren A, Kofod H, Holst JJ (1994) Tissue and plasma concentrations of amidated and glycine-extended glucagon-like peptide I in humans. Diabetes 43:535-539

34. Lowry CV, Kimmey JS, Felder S et al (1978) Enzyme patterns in single human muscle fibers. J Biol Chem 253:8269-8277

35. Van CE, Mestrez F, Sturis J, Polonsky KS (1992) Estimation of insulin secretion rates from C-peptide levels. Comparison of individual and standard kinetic parameters for C-peptide clearance. Diabetes 41:368-377

36. Meneilly GS, Elliott T (1998) Assessment of insulin sensitivity in older adults using the hyperglycemic clamp technique. J Am Geriatr Soc 46:88-91

37. Tudor-Locke C, Bell RC, Myers AM et al (2004) Controlled outcome evaluation of the First Step Program: a daily physical activity intervention for individuals with type II diabetes. Int J Obes Relat Metab Disord 28:113-119

38. Mitranun W, Deerochanawong C, Tanaka H, Suksom D (2013) Continuous vs interval training on glycemic control and macro- and microvascular reactivity in type 2 diabetic patients. Scand J Med Sci Sports

39. Albu JB, Heilbronn LK, Kelley DE et al (2010) Metabolic changes following a 1-year diet and exercise intervention in patients with type 2 diabetes. Diabetes 59:627-633

40. Ross R, Dagnone D, Jones PJ et al (2000) Reduction in obesity and related comorbid conditions after diet-induced weight loss or exercise-induced weight loss in men. A randomised, controlled trial. Ann Intern Med 133:92-103

41. Vind BF, Pehmoller C, Treebak JT et al (2011) Impaired insulininduced site-specific phosphorylation of TBC1 domain family, member 4 (TBC1D4) in skeletal muscle of type 2 diabetes patients is restored by endurance exercise-training. Diabetologia 54:157-167

42. Holloszy JO (2005) Exercise-induced increase in muscle insulin sensitivity. J Appl Physiol 99:338-343

43. Sano H, Kane S, Sano E et al (2003) Insulin-stimulated phosphorylation of a Rab GTPase-activating protein regulates GLUT4 translocation. J Biol Chem 278:14599-14602

44. Mitrakou A, Vuorinen-Markkola H, Raptis G et al (1992) Simultaneous assessment of insulin secretion and insulin sensitivity using a hyperglycemia clamp. J Clin Endocrinol Metab 75:379-382

45. Nishida Y, Tokuyama K, Nagasaka S et al (2004) Effect of moderate exercise training on peripheral glucose effectiveness, insulin sensitivity, and endogenous glucose production in healthy humans 
estimated by a two-compartment-labeled minimal model. Diabetes 53:315-320

46. Solomon TP, Malin SK, Karstoft K, Haus JM, Kirwan JP (2013) The influence of hyperglycemia on the therapeutic effect of exercise on glycemic control in patients with type 2 diabetes mellitus. JAMA Intern Med 173:1834-1836

47. Hojlund K, Staehr P, Hansen BF et al (2003) Increased phosphorylation of skeletal muscle glycogen synthase at NH2-terminal sites during physiological hyperinsulinemia in type 2 diabetes. Diabetes 52:1393-1402
48. Dela F, Larsen JJ, Mikines KJ, Ploug T, Petersen LN, Galbo H (1995) Insulin-stimulated muscle glucose clearance in patients with NIDDM. Effects of one-legged physical training. Diabetes 44: $1010-1020$

49. Phielix E, Meex R, Moonen-Kornips E, Hesselink MK, Schrauwen $P$ (2010) Exercise training increases mitochondrial content and ex vivo mitochondrial function similarly in patients with type 2 diabetes and in control individuals. Diabetologia 53:1714-1721

50. Hoeks J, Schrauwen P (2012) Muscle mitochondria and insulin resistance: a human perspective. Trends Endocrinol Metab 23:444-450 